\title{
Propiedades psicométricas del TAPQOL 2 para la medición de la calidad de vida en niños preescolares colombianos*
}

\section{Psychometric Properties of the TAPQOL 2 to Measure of Quality of Life in Colombian Preschool Children}

\author{
Stefano Vinaccia Alpi* \\ Universidad del Sinú, Montería, Colombia \\ Andrés Gómez-Acosta \\ ORCID: 0000-0002-7932-0466 \\ Fundación Universitaria Sanitas, Bogotá, \\ Colombia \\ Rosangela Raleigh \\ Universidad del Sinú, Montería, Colombia
}

Alfonso Urzúa

Universidad Católica del Norte Antofagasta,

Chile

\section{Andrea Ortega}

Alejandra Vieria, Universidad del Sinú, Montería, Colombia

Alejandra Díaz

ORCID: 0000-0003-3405-3023

Alexander González

ORCID: 0000-0002-8413-4123

Fundación Universitaria Sanitas, Bogotá,

Colombia

Yuli Jiménez

Lina Pico

Brayan Vinasco

Universidad del Sinú, Montería, Colombia

Recibido: 22 de junio de 2019

Revisado: 7 de agosto de 2019

Aceptado: 22 de octubre de 2019

\section{Resumen}

Si bien hay antecedentes empíricos acerca de la medición de la calidad de vida relacionada con la salud (CVRS) en niños, no hay evidencia de estudios similares en Colombia. Por tal motivo, el objetivo de la investigación fue describir las propiedades psicométricas iniciales de la versión en español del TAPQOL 2 en una muestra de 229 cuidadores de niños preescolares entre tres y seis años de edad, de ambos sexos, residentes en el departamento de Córdoba, Colombia. Se realizó un análisis factorial con extracción de componentes principales y rotación Varimax, así como un análisis de consistencia interna por alfa de Cronbach para la escala general y cada factor obtenido, correlaciones inter-elemento y estadísticos total-elemento. Los resultados mostraron que el cuestionario TAPQOL 2 presenta un alfa de Cronbach de 0.81 , explica una varianza acumulada del $66.44 \%$ y correlaciones positivas entre todos los reactivos. Con relación al instrumento original, el cuestionario TAPQOL 2 mantiene 12 factores, pero se diferencia en que se fusionan los factores emociones positivas y vitalidad, mientras que emerge un nuevo factor relacionado con el control comportamental del niño. Se encuentra que los indicadores psicométricos del cuestionario son consistentes con investigaciones previas sobre los componentes del bienestar en los niños.

Palabras clave: TAPQOL 2; bienestar psicológico; propiedades psicométricas; niñez.

Artículo de Investigación. Citar como: Vinaccia, A. S., Gómez-Acosta, A., Raleigh, R. Urzúa, A., Ortega, A. ... Vinasco, B. (2020). Propiedades psicométricas del TAPQOL 2 para la medición de la calidad de vida en niños preescolares colombianos. Diversitas: Perspectivas en Psicología, 16(1), 13-24. Dol: https://doi.org/10.15332/22563067.5538

** Autor de correspondencia: Stefano Vinaccia Alpi, Ph. D. Programa de Psicología, Universidad del Sinú. Dirección postal: Carrera 1w 38-153, Montería, Colombia. Correo electrónico: vinalpi47@hotmail.com; Stefanovinacci@unisinu.edu.co. 


\section{Abstract}

While there are empirical antecedents in the measurement of Health-Related Quality of Life (HRQOL) in children, there is no evidence of similar studies in Colombia. For this reason, the objective of this investigation is to describe the initial psychometric properties of the Spanish version of the TAPQOL 2 in a sample of 229 caregivers for preschool children of both sexes between three and six years of age who reside in the department of Córdoba, Colombia. A factorial analysis was carried out with extraction of the main components and Varimax rotation, as well as internal consistency analysis by means of the Cronbach's alpha coefficient for the general scale and for every obtained factor, interelement correlations and total-element statistics. Results showed that the TAPQOL 2 questionnaire presents a Cronbach's alpha of 0.81 , explains a cumulative variance of $66.44 \%$, and presents positive correlations across all items. In relation to the original instrument, the TAPQOL 2 questionnaire keeps 12 factors, but it differs in that the positive emotions and vitality factors are merged in the same factor, while a new factor related to the behavioral control of the child emerges. It was found that the psychometric indicators of the questionnaire are consistent with previous research on the components of child wellbeing.

Keywords: TAPQOL 2; psychological well-being; psychometric properties; childhood.

\section{Introducción}

De acuerdo con The World Health Organization Quality of life assessment (1995), la calidad de vida consiste en la percepción que posee el individuo sobre su posición en la vida dentro del contexto cultural y el sistema de valores en el que vive y con respecto a sus metas, expectativas, normas y preocupaciones. Específicamente en el caso de los niños, Quiceno y Vinaccia (2013) define la calidad de vida infantil (cvI) como "la percepción del bienestar físico, psicológico y social del niño(a) y adolescente dentro de un contexto cultural específico de acuerdo a su desarrollo evolutivo y sus diferencias individuales" (p. 77). Mientras la calidad de vida relacionada con la salud infantil [CVRSI] sería "la valoración que tiene el niño(a) y adolescente de acuerdo con su desarrollo evolutivo, sus diferencias individuales y su contexto cultural de su funcionamiento físico, psicológico y social cuando este ha sido limitado o alterado por la presencia de una enfermedad o accidente" (Quiceno, 2013, p.77).

Los estudios de medición de la CVRS en la infancia y la adolescencia se empiezan a vislumbrar desde los años 60 (Quiceno \& Vinaccia, 2008); no obstante, desde los años 90 se ha contado con un paulatino aumento de investigaciones en el área, ya sea 
para la validación de pruebas o estudios que han buscado analizar las relaciones de algunas patologías con este constructo. Se ha encontrado en general una percepción desfavorable de cVRS de adolescentes y adultos que presentaban alguna patología física, en comparación con muestras sanas (Quiceno \& Vinaccia, 2008, 2013; Vinaccia \& Quiceno, 2010, 2012).

El diseño y validación de instrumentos de medida en CVRSI han cobrado mayor relevancia (Rajmil, et al., 2011) apoyados en los avances técnicos y teóricos de la psicometría (Aymerich et al., 2007). La mayoría de dichas escalas y cuestionarios son de origen anglosajón (Aymerich et al., 2007; Bullinger et al., 1998) y focalizados desde una perspectiva biomédica. Es decir, centrados en los síntomas y signos de la enfermedad más que en la valoración subjetiva del funcionamiento y bienestar personal del niño o adolescente (Urzúa et al., 2009). Adicional a esto, se encuentra la complejidad para medir la cVRS de niños pre-escolares por la ausencia de instrumentos específicos para estos segmentos de edad. Uno de los instrumentos más conocido es el cuestionario TAPQOL (Preschool Children Quality of Life) el cual, aborda una descripción del nivel de salud en niños preescolares evaluándola desde las diferentes áreas de desarrollo por medio de una prueba multidimensional (Bunge et al., 2005).

Esta prueba psicométrica consta de 43 ítems incluidos en 12 subdimensiones, basándose y evaluando los tipos de funcionamiento físico, emocional y social. La utilidad de la aplicación del instrumento radica en que los datos se convierten en información veraz para el proceso de toma de decisiones en materia diagnóstica de pacientes en edad preescolar, no solo para el ámbito terapéutico sino también para el diseño de estrategias de abordaje promocional y preventivo de tales situaciones por parte del sector salud y otras instituciones implicadas (Fekkes et al., 2000; Rajmil et al., 2011). Este instrumento ha sido validado en países como Brasil (Benini, 2014), Chile (Urzúa et al., 2010), Malasia (Tay, Jalaludin, Low, \& Lim, 2015), España (Sardón-Prado et al., 2008), China (Lee et al., 2005) y Holanda (Schepers et al., 2017).

Si bien en Colombia se han validado diferentes instrumentos para medir la cVRS en niños y adolescentes como el Kidscreen 27 (Quintero et al., 2012), Kidscreen 52 (Jaimes, 2008), PEdsqol4.0 (Vélez et al., 2016), y el EQ-5D-Y (Gaitán, Correa, Vinaccia, \& Ramírez, 2017), aun se presenta un vacío relacionado con la medición de la cVRs en menores de 14 años. Por otra parte, contar con un instrumento especializado para tal tipo de población permitiría medir la CVRs tanto en las grandes urbes del país, como en regiones apartadas y marginadas de Colombia como la Costa Atlántica, los Llanos Orientales y la Amazonía, donde dicha población supera un $25 \%$ del total habitantes censados (Departamento Nacional de Estadística [DANE], 2019). Por lo tanto, el presente estudio de campo tiene como objetivo evaluar las propiedades psicométricas de la versión en español del cuestionario TZO-AzL sobre la calidad de vida de los niños en edad preescolar (TAPQOL), y determinar su utilidad en el contexto colombiano.

\section{Método}

\section{Diseño}

Corresponde a una investigación empírico - analítica, de corte psicométrico.

\section{Participantes}

Se obtuvo una muestra estratificada correspondiente a 229 cuidadores con niños ( 110 mujeres) en edades entre tres y seis años, obtenidos aleatoriamente de dos centros educativos. En la tabla 1 se reportan las características de los participantes. 
Tabla 1.

Información sociodemográfica de la muestra

\begin{tabular}{|c|c|c|}
\hline Estado Civil Cuidador & $\mathrm{n}$ & $\%$ \\
\hline Soltero & 23 & 10.1 \\
\hline Casado & 76 & 33.5 \\
\hline Separado & 8 & 3.5 \\
\hline Unión Libre & 117 & 51.5 \\
\hline Viudo & 3 & 1.3 \\
\hline Nivel de estudios Cuidador & $\mathrm{n}$ & $\%$ \\
\hline Primaria & 23 & 10.1 \\
\hline Secundaria & 73 & 32.2 \\
\hline Tecnología & 63 & 27.8 \\
\hline Profesional & 36 & 15.9 \\
\hline Posgrado & 32 & 14.1 \\
\hline Estrato Socioeconómico & $\mathrm{n}$ & $\%$ \\
\hline 1 & 132 & 57.6 \\
\hline 2 & 47 & 20.5 \\
\hline 3 & 10 & 4.4 \\
\hline 4 & 15 & 6.6 \\
\hline 5 & 11 & 4.8 \\
\hline 6 & 14 & 6.1 \\
\hline
\end{tabular}

Fuente: elaboración propia.

\section{Instrumento}

El TAPQOL (Preschool Children Quality of Life Questionare) es un instrumento heteroaplicado diseñado en Holanda por Fekkes et al., (2000), y reportó en su versión original coeficientes alfa de Cronbach superiores a 0.83 en la escala total (Urzúa et al., 2010) y engloba tres dimensiones: funcionamiento físico (F1), funcionamiento social (F2), y funcionamiento emocional (F3) a su vez integrados por 12 subdimensiones. Consta de 43 ítems distribuidos en los tres factores mencionados. La escala plantea que se contestan únicamente las primeras 32 preguntas si el niño tiene menos de 18 meses, y la totalidad de la encuesta si los niños superan la edad mencionada.

\begin{tabular}{lcc}
\hline \multicolumn{1}{c}{ Sexo del cuidador } & $\mathrm{n}$ & $\%$ \\
\hline Hombre & 28 & 12.2 \\
Mujer & 201 & 87.2 \\
\hline
\end{tabular}

\begin{tabular}{lcc}
\hline \multicolumn{1}{c}{ Sexo del niño } & $\mathrm{n}$ & $\%$ \\
\hline Hombre & 119 & 52 \\
Mujer & 110 & 48 \\
\hline
\end{tabular}

\begin{tabular}{ccc}
\hline Edad del niño & $\mathrm{n}$ & $\%$ \\
\hline 3 & 38 & 16.59 \\
4 & 72 & 31.44 \\
5 & 79 & 34.50 \\
6 & 40 & 17.47 \\
\hline
\end{tabular}

\begin{tabular}{lcc}
\hline Sistema de Salud Adscrito & $\mathrm{n}$ & $\%$ \\
\hline SISBEN (público) & 104 & 45.4 \\
EPS & 85 & 37.1 \\
Medicina Prepagada & 37 & 16.2 \\
Particular & 3 & 1.3 \\
\hline
\end{tabular}

En principio se implementan las preguntas orientadas a identificar limitaciones funcionales ocurridas en los tres últimos meses, para finalizar con las reacciones que han podido tener dichos niños ante dicha situación.

\section{Procedimiento}

Inicialmente se establecieron las alianzas respectivas con los centros educativos elegidos $(n=2)$, para proceder a la socialización del estudio con los participantes y a la obtención de los correspondientes avales. Posterior a esto se recolectó la información, con la verificación de diligenciado completo, evitando omitir información. Para la toma de la 
muestra se tuvo en cuenta las consideraciones éticas respectivas para la investigación con sujetos humanos en Colombia (Ministerio de Salud, 1993; Congreso de Colombia, 2006), en particular con los cuidadores informantes.

\section{Análisis de datos}

Los datos obtenidos se analizaron con el software SPSS $24{ }^{\circledR}$, realizando inicialmente una descripción de la muestra, para enseguida proyectar un análisis factorial exploratorio con extracción de mínimos cuadrados no ponderados y rotación Varimax. Se establecieron los índices de confiabilidad con alfa de Cronbach, y se revisó la consistencia interna a partir de una matriz de correlaciones.

\section{Resultados}

Para determinar si el tamaño muestral al que se acudió fue suficiente para proceder con el análisis factorial exploratorio, se calcularon los correspondientes coeficientes Kaiser-Meyer-Olkin (кмо) y prueba de esfericidad de Bartlett, los cuáles se presentan en la tabla 2.

Tabla 2.

Prueba de adecuación y suficiencia muestral

\begin{tabular}{lcc}
\hline $\begin{array}{l}\text { Medida Kaiser-Meyer-Olkin de adecuación } \\
\text { de muestreo }\end{array}$ & 0.767 \\
\hline Prueba de esfericidad & Aprox. Chi-cuadrado & 4197 \\
de Bartlett & gl & 903 \\
& Sig. & .00 \\
\hline
\end{tabular}

Fuente: elaboración propia.

En relación con lo expuesto en la tabla 2, se encontró que las variables están intercorrelacionadas de manera adecuada para aplicar el mismo tipo de análisis realizado en la muestra española (i. e., componentes principales con rotación Varimax). Así, se encontró que la prueba con muestra colombiana explica el $66.44 \%$ de la varianza total, por lo cual resulta un instrumento idóneo para la medición del bienestar psicológico en niños colombianos. Este análisis se ha realizado sobre las puntuaciones estandarizadas de cada dimensión (Tabla 3).

Tabla 3.

Matriz de cargas vectoriales, carga factorial de cada reactivo y alfa de Cronbach si se suprime el elemento del TAPQOL 2

\begin{tabular}{|c|c|c|c|c|c|c|c|c|c|c|c|c|c|c|c|c|}
\hline Ítem & 1 & 2 & 3 & 4 & 5 & 6 & 7 & 8 & 9 & 10 & 11 & 12 & A & B & C & $D$ \\
\hline 1 & 0.72 & & & & & & & & & & & & 0.30 & 48.44 & 0.85 & 0.81 \\
\hline 2 & 0.48 & & & & & & & & & & & & 0.33 & 49.15 & 0.58 & 0.81 \\
\hline 3 & & 0.67 & & & & & & & & & & & 0.42 & 49.24 & 0.58 & 0.81 \\
\hline 4 & & 0.73 & & & & & & & & & & & 0.37 & 47.74 & 0.83 & 0.81 \\
\hline 5 & & 0.67 & & & & & & & & & & & 0.48 & 48.18 & 0.68 & 0.81 \\
\hline 6 & & & 0.62 & & & & & & & & & & 0.38 & 49.24 & 0.65 & 0.81 \\
\hline 7 & & & 0.76 & & & & & & & & & & 0.67 & 48.02 & 0.87 & 0.81 \\
\hline 8 & & & 0.66 & & & & & & & & & & 0.33 & 49.42 & 0.50 & 0.81 \\
\hline 9 & 0.59 & & & & & & & & & & & & 0.15 & 48.86 & 0.61 & 0.81 \\
\hline 10 & & & & 0.69 & & & & & & & & & 0.42 & 46.95 & 0.77 & 0.80 \\
\hline 11 & & & & 0.61 & & & & & & & & & 0.45 & 47.90 & 0.63 & 0.81 \\
\hline 12 & & & & 0.70 & & & & & & & & & 0.45 & 47.29 & 0.75 & 0.80 \\
\hline 13 & & & & 0.68 & & & & & & & & & 0.39 & 48.03 & 0.59 & 0.81 \\
\hline 14 & & & & & 0.83 & & & & & & & & 0.69 & 46.56 & 0.88 & 0.80 \\
\hline 15 & & & & & 0.84 & & & & & & & & 0.77 & 46.04 & 0.88 & 0.80 \\
\hline 16 & & & & & 0.81 & & & & & & & & 0.69 & 46.28 & 0.87 & 0.80 \\
\hline
\end{tabular}




\begin{tabular}{|c|c|c|c|c|c|c|c|c|c|c|c|c|c|c|c|c|}
\hline Ítem & 1 & 2 & 3 & 4 & 5 & 6 & 7 & 8 & 9 & 10 & 11 & 12 & A & $B$ & $C$ & $\mathrm{D}$ \\
\hline 17 & & & & & & 0.79 & & & & & & & 0.50 & 47.12 & 0.66 & 0.80 \\
\hline 18 & & & & & & 0.64 & & & & & & & 0.52 & 46.61 & 0.72 & 0.80 \\
\hline 19 & & & & & & 0.65 & & & & & & & 0.53 & 46.01 & 0.76 & 0.80 \\
\hline 20 & & & & & & 0.70 & & & & & & & 0.44 & 46.69 & 0.72 & 0.80 \\
\hline 21 & & & & & & 0.48 & & & & & & & 0.51 & 45.90 & 0.71 & 0.80 \\
\hline 22 & & & & & & 0.60 & & & & & & & 0.54 & 46.25 & 0.73 & 0.80 \\
\hline 23 & & & & & & & 0.82 & & & & & & 0.17 & 48.39 & 1.00 & 0.81 \\
\hline 24 & & & & & & & & 0.67 & & & & & 0.73 & 50.74 & 0.72 & 0.82 \\
\hline 25 & & & & & & & & 0.75 & & & & & 0.77 & 50.19 & 0.69 & 0.81 \\
\hline 26 & & & & & & & & 0.76 & & & & & 0.75 & 50.38 & 0.71 & 0.82 \\
\hline 27 & & & & & & & & & 0.47 & & & & 0.27 & 46.95 & 0.73 & 0.80 \\
\hline 28 & & & & & & & & & 0.78 & & & & 0.68 & 47.02 & 0.76 & 0.80 \\
\hline 29 & & & & & & & & & 0.74 & & & & 0.40 & 46.71 & 0.77 & 0.80 \\
\hline 30 & & & & & & & & 0.68 & & & & & 0.34 & 49.59 & 0.74 & 0.81 \\
\hline 31 & & & & & & & & 0.74 & & & & & 0.54 & 49.70 & 0.63 & 0.81 \\
\hline 32 & & & & & & & & 0.85 & & & & & 1.00 & 49.86 & 0.66 & 0.81 \\
\hline 33 & & & & & & & & & & 0.80 & & & 0.56 & 50.28 & 0.75 & 0.81 \\
\hline 34 & & & & & & & & & & 0.78 & & & 0.56 & 50.19 & 0.75 & 0.81 \\
\hline 35 & & & & & & & & & & 0.79 & & & 0.67 & 50.44 & 0.82 & 0.82 \\
\hline 36 & & & & & & & & & & & 0.83 & & 0.66 & 49.62 & 0.50 & 0.81 \\
\hline 37 & & & & & & & & & & & 0.82 & & 1.00 & 49.40 & 0.79 & 0.81 \\
\hline 38 & & & & & & & & & & & 0.87 & & 0.78 & 49.88 & 0.61 & 0.81 \\
\hline 39 & & & & & & & & & & & 0.89 & & 0.89 & 49.86 & 0.71 & 0.81 \\
\hline 40 & & & & & & & & & & & & 0.63 & 0.41 & 49.26 & 0.65 & 0.81 \\
\hline 41 & & & & & & & & & & & & 0.73 & 0.46 & 48.46 & 0.71 & 0.81 \\
\hline 42 & & & & & & & & & & & & 0.80 & 0.60 & 48.39 & 0.79 & 0.81 \\
\hline 43 & & & & & & & & & & & & 0.79 & 0.61 & 48.35 & 0.75 & 0.81 \\
\hline
\end{tabular}

Convenciones: Factor 1: Problemas digestivos. Factor 2: Problemas en la piel. Factor 3: Problemas respiratorios. Factor 4: Problemas del sueño. Factor 5: Problemas con apetito. Factor 6: Problemas de conducta. Factor 7: Control del hijo. Factor 8:. Emociones positivas y vitalidad (se fusionan en un solo factor de acuerdo al peso en la varianza demostrado en el análisis) Factor 9: Problemas de ansiedad. Factor 10: Funcionamiento social. Factor 11: Funcionamiento motor. Factor 12: Dificultades de cognición - comunicación. A. = Extracción. B. = Varianza de escala si el elemento se ha suprimido. $C$. = Correlación ítem - dimensión específica a la que se ajusta. D. = Alfa de Cronbach si el elemento se ha suprimido.

Fuente: elaboración propia.

En relación con la tabla 3 , se evidenció que existen correlaciones entre cada ítem y el factor al cual pertenece por encima del .50, recomendado por Hair, Black, Babin y Anderson (2014) como punto de referencia para garantizar una mayor validez discriminante de cada factor, y que el alfa de Cronbach del total de la prueba varía muy poco si se elimina alguno de los elementos.
Por otro lado, la tabla 4, compara los coeficientes de confiabilidad obtenidos en la muestra de niños colombianos por cada factor. Se puede observar que las dimensiones con coeficiente alfa menores a 0.70 fueron: tracto digestivo (0.52). piel (0.61). tracto respiratorio (0.58) y ansiedad (0.67), al igual que en social (0.69), aspecto que deberá ser discutido y superado. 
Tabla 4. Comparativo de las propiedades psicométricas de fiabilidad del cuestionario de TAPQOL 2

\begin{tabular}{lcccccccc}
\hline & $\begin{array}{c}\mathbf{N}^{\circ} \\
\text { Factor }\end{array}$ & Mín & Máx & Media & $\begin{array}{c}\text { Desviación } \\
\text { Típica }\end{array}$ & $\begin{array}{c}\text { Varianza } \\
\text { Explicada }\end{array}$ & Alfa & $\begin{array}{c}\text { Diferencia } \\
\text { de } \\
\text { medianas } \\
\text { por sexo }\end{array}$ \\
\hline F1. Tracto digestivo & 3 & 3 & 8 & 4.08 & 1.00 & 3.54 & 0.52 & .64 \\
F2. Piel & 3 & 3 & 9 & 3.94 & 1.28 & 4.46 & 0.61 & .63 \\
F3. Tracto respiratorio & 3 & 3 & 8 & 3.49 & 0.93 & 4.27 & 0.58 & $.04^{*}$ \\
F4. Sueño & 4 & 4 & 12 & 5.14 & 1.46 & 5.48 & 0.72 & .19 \\
F5. Apetito & 3 & 3 & 9 & 4.70 & 1.63 & 5.89 & 0.86 & .80 \\
F6. Problemas de conducta & 6 & 6 & 18 & 9.79 & 2.53 & 7.24 & 0.82 & $.01^{*}$ \\
F7. Control del niño & 1 & 1 & 3 & 1.36 & 0.58 & 2.85 & --- & .94 \\
F8. Emociones positivas y vitalidad & 3 & 6 & 18 & 16.93 & 1.87 & 8.50 & 0.86 & .18 \\
F9. Ansiedad & 3 & 3 & 9 & 4.61 & 1.37 & 5.16 & 0.67 & .24 \\
F10. Funcionamiento social & 3 & 3 & 9 & 8.53 & 1.01 & 5.62 & 0.8 & .40 \\
F11. Funcionamiento motor & 4 & 4 & 16 & 4.17 & 0.92 & 7.57 & 0.85 & .52 \\
F12. Cognición - comunicación & 4 & 4 & 12 & 5.13 & 1.53 & 5.82 & 0.76 & .41 \\
\hline Total TApQoL & 43 & 56 & 94 & 71.87 & 7.10 & 66.44 & 0.81 & .06 \\
\hline
\end{tabular}

Nota: * Significación estadística al 0.05

Fuente: elaboración propia.

De acuerdo con la tabla 4 solo se evidencian diferencias significativas entre niños y niñas en los factores de problemas en tracto respiratorio y de problemas de comportamiento reportados, lo cual plantea que en diez de las doce categorías las diferencias no se explicarían en función al sexo de los niños. 


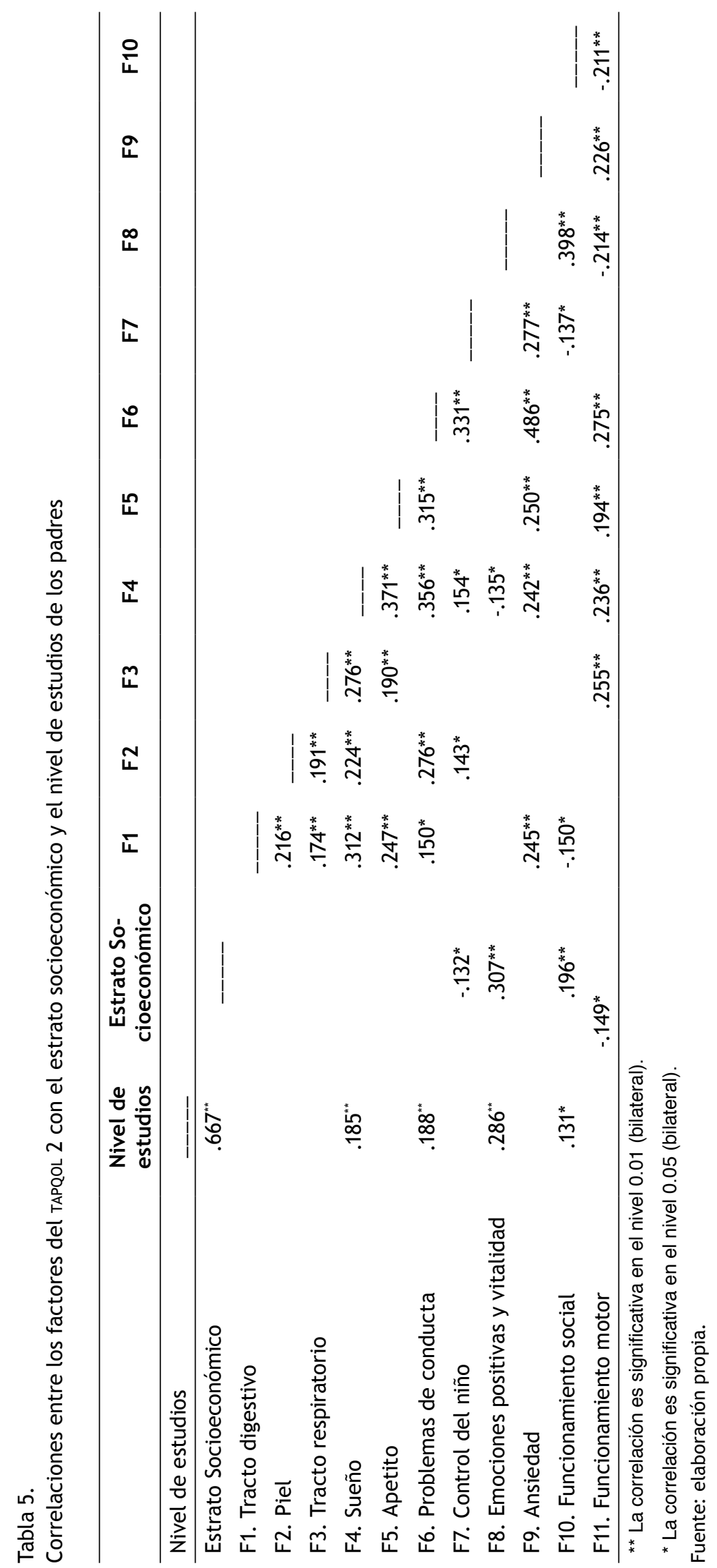


En relación con la tabla 5 , se identificó que el factor 12 (relacionado con dificultades en cognición y comunicación) no se correlacionó con ninguna de las demás dimensiones relacionadas, y que la correlación entre ansiedad y problemas de conducta es alta; las otras dimensiones mostraron correlaciones bajas, pero con significación estadística, lo cual es esperable desde la evidencia empírica.

\section{Discusión}

Los resultados del instrumento TAPQOL Como método para identificar la CVRSI en función de la sintomatología de enfermedades que padecen los niños, permiten acopiar criterios válidos para implementar alternativas preventivas en el contexto de la atención primaria dirigidos a atender las condiciones desencadenantes, y por esta vía reducir los síntomas físicos, emocionales y sociales que afectan la CVRSI. Sin embargo, de acuerdo con Urzúa et al. (2010), es necesario que el instrumento mantenga la suficiente sensibilidad en relación con características propias, tanto de la cultura como del contexto físico que determinan estilos de vida.

Como se pudo observar en los resultados, la forma de agrupación de los ítems reportado en los resultados difiere un poco con relación con la versión original presentada por Bunge et al. (2005), dado que el factor 8 fusiona los ítems de los factores emociones positivas y vitalidad; mientras, el ítem 23 (no ha podido controlar a su hijo), no se agrupó con ninguno de los demás factores, y aun cuando correlacionó en gran medida con el factor 9 (ansiedad) constituye un factor único que se decide mantener como dimensión independiente (problemas de conducta), teniendo en cuenta que una mayor presencia de dificultades en el comportamiento puede representar mayor tendencia a presentar problemas de atención, aprendizaje, dificultades en estados de ánimo y adaptabilidad (Rodríguez, 2010), que a su vez pueden comprender la CVRSI (Quiceno \& Vinaccia, 2008, 2013).

De igual manera, las correlaciones significativas que se encontraron entre los factores agrupados son de baja magnitud, hecho que era esperable, teniendo en cuenta que cada factor mide aspectos de la cVRs diferentes pero complementarios entre sí, por lo cual se descarta colinealidad en la escala evaluada, de acuerdo con los supuestos de Pérez y Medrano (2010). En esta misma línea se demuestra que, tanto el nivel de estudios como la formación académica del cuidador, se relacionan como factores protectores de la presencia de problemas del sueño, conducta, mayor cantidad de emociones positivas y funcionamiento social (nivel de estudios), y más emociones positivas y funcionamiento social (posición socioeconómica). Sin embargo, existen algunas correlaciones negativas llamativas entre el funcionamiento motor, las emociones negativas y el funcionamiento social, y entre el estrato socioeconómico y el control del niño, aspectos que coinciden con lo encontrado por Aguirre-Dávila (2015) en términos de la evitación al daño manifestada por los niños de nivel socioeconómico más bajo, pero que deben evaluarse a futuro con mayor detalle para una mejor comprensión y formulación de acciones en salud al respecto (Piqueras, Ramos, Martínez, \& Oblitas, 2009).

Por otro lado, se evidencia que tanto los problemas asociados al tracto respiratorio, como los problemas de comportamiento, son los únicos que presentan diferencias significativas en función del sexo de los niños; esto puede estar relacionado con la propensión de las niñas a tener mayor control inhibitorio en relación con los niños de la misma edad (Reyna \& Brussino, 2015), y con la tendencia a que existan diferencias en la presentación de síntomas de infecciones respiratorias agudas entre ambos sexos (Ferreira-Guerrero et al., 2013). Aunque esto último también puede estar condicionado a covariables como la edad (más frecuente en menores de cinco años) y la zona donde se desenvuelven cotidianamente los niños, con mayor tendencia a dichas enfermedades en zonas urbanas, húmedas de clima variable y con alta contaminación del aire (Domínguez, Tapia, Hernández, \& Castillo, 2017).

Si bien se encuentra que el TAPQOL es instrumento válido y confiable para la medición de la cVRSI, es importante continuar con la validación de instrumentos que permitan reconocer la percepción del bienestar y calidad de vida en función en la etapa de desarrollo que se encuentre la población, que a su vez sean sensibles a aspectos como el nivel de escolarización, la crianza en condiciones ambien- 
tales particulares (rural-urbano), y la red de apoyo familiar, entre otros aspectos (Gaitán et al., 2017).

En conclusión, tanto las investigaciones como las intervenciones preventivas en salud para población infantil y adolescente deben contar con instrumentos de evaluación para el cVRSI. De acuerdo con los resultados referidos (correlación ítem - factor, validez discriminante y alfa de (ronbach) se demuestra que el TAPQOL es un instrumento idóneo para medir dicha variable psicosocial en población colombiana. En ese ámbito, se recomienda el uso de este tipo de escalas con los profesionales que atienden a esta población, así con quienes desarrollan estudios frente al tema en el contexto colombiano.

\section{Referencias}

Aguirre-Dávila, E. (2015). Prácticas de crianza, temperamento y comportamiento prosocial de estudiantes de educación básica. Revista Latinoamericana de Ciencias Sociales, Niñez y Juventud, 13 (1), 223-243.

Aymerich, M., Berra, S., Guillamón, I., Herdman, M., Alonso, J. ... Rajmil, L. (2007). Desarrollo de la versión en español del Kidscreen, un cuestionario de calidad de vida para la población infantil y adolescente. Gaceta Sanitaria, 19(2), 93-102.

Benini, C. (2014). Validação linguística e cultural da versão brasileira do TNO-AZL Preschool Children's Quality of Life questionnaire(TAPQOL) para crianças em idade préescolar/. Porto Alegre: PUCRS.

Bullinger, M., Alonso, J., Apolone, G., Leplege, A., Sulli-van, M., ... Wake, J. E. (1998). Translating health status questionnaires and evaluating their quality: the IQOLA project approach. International quality of life assessment. Journal of Clinical Epidemiology, 51(11), 913-923.

Bunge, E., Essink-Bot, M., Kobussen, M., van Suijlekom-Smit, L., Moll, H., \& Raat, H. (2005). Reliabilitry and validity of health status measurement by the TAPQOL. Archives of Disease in Childhood, 90(4), 351-358.
Congreso de Colombia. (2006). Ley 1090 "por la cual se reglamenta el ejercicio de la profesión de Psicología, se dicta el Código Deontológico y Bioético y otras disposiciones". Recuperado de http://www.funcionpublica.gov.co/eva/gestornormativo/norma_pdf.php?i=66205

Departamento Nacional de Estadística [DANE] (2019). Censo Nacional de Población y Vivienda 2018. Recuperado de: https://www.dane. gov.co/index.php/estadisticas-por-tema/demografia-y-poblacion/censo-nacional-depoblacion-y-vivenda-2018/donde-estamos/ mapas-tematicos

Domínguez, R., Tapia, E., Hernández, J., \& Castillo, I. (2017). Edad y nivel educativo asociados al conocimiento sobre signos de alarma para infecciones respiratorias en madres adolescentes. Revista Cuidarte, 8(2), 1628-1637. https:// doi.org/10.15649/cuidarte.v8i2.395

Fekkes, M., Theunissen, N. C. M., Brugman, E., Veen, S., Verrips, E. G., ... Verloove-Vanhorick, S. P. (2000). Development and psychometric evaluation of the TAPQOL: A health-related quality of life instrument for 1-5-year-old children. Quality of Life, 9(8), 961-972.

Ferreira-Guerrero, E., Báez-Saldaña, R., TrejoValdivia, B., Ferreyra-Reyes, L., Delgado Sánchez, G., ... García-García, L. (2013). Infecciones respiratorias agudas en niños y signos de alarma identificados por padres y cuidadores en México. Salud Pública de México, 55(2), 307-313.

Gaitán, D., Correa, J., Vinaccia, S., \& Ramírez, R. (2017). Auto reporte de la calidad de vida de niños y adolescentes colombianos: Estudio FuPRECOL. Colombia Medica, 48(1), 12-18.

Hair, J., Black, W., Babin, B., \& Anderson, R. (2014). Multivariate Data Analysis (Seventh edition). London: Pearson Education Limited.

Jaimes, M. L. (2008). Adaptación cultural a la población escolar colombiana del KIDSCREEN y del VSP-A, 
instrumentos de calidad de vida relacionada con la salud (CVRS) para niños(as) y adolescentes (Tesis doctoral). Universidad de Alicante, Alicante, España.

Lee, C. F., Chien, L. Y., Ko, Y. L., Chou, Y. H., Thai, C. J., \& Liou, Y. M. (2005). Development and psychometric properties of the Chinese language version of the tapqol: a health-related quality of life instrument for preschool children. International Journal Nursering Studies, 42(4), 457-465.

Ministerio de Salud. (1993). Resolución 8430, “por la cual establecen las normas científicas, técnicas y administrativas para la investigación en salud". Recuperado de https://www.minsalud. gov.co/sites/rid/Lists/BibliotecaDigital/RIDE/ DE/DIJ/RESOLUCION-8430-DE-1993.PDF

Pérez, E. \& Medrano, L. (2010). Análisis factorial exploratorio: bases conceptuales y metodológicas. Revista Argentina de Ciencias del Comportamiento, 2(1), 58-66.

Piqueras, J., Ramos, V., Martínez, A., \& Oblitas, L. (2009). Emociones negativas y su impacto en la salud mental y física. Suma Psicológica, 16(2), 85-112.

Quiceno, J. M. \& Vinaccia, S. (2008). Calidad de vida relacionada con la salud infantil: Una aproximación conceptual. Psicología y Salud, 18(1), 33-44.

Quiceno, J. M. \& Vinaccia, S. (2013). Calidad de vida relacionada con la salud en niños y adolescentes con enfermedad crónica. Psychologia: Avances en la Disciplina, 7(2), 69-86.

Quintero, C. A., Lugo, L. E., García, H. I., \& Sánchez, A. (2011). Validación del cuestionario KIDSCREEN-27 de calidad de vida relacionada con la salud en niños y adolescentes de Medellín, Colombia. Revista Colombiana de Psiquiatría, 40(3), 470-487.

Rajmil, L., Abad, S., Sardon, O., Morera G, PérezYarza E. G., .... Alonso, J. (2011). Reliability and validity of the Spanish version of the TAPQOL: a health-related quality of life (HRQOL) instrument for 1-to 5-year-old children. International Journal of Nursing Studies, 48(5), 549-56. http://doi.org/10.1016/j.ijnurstu.2010.09.004

Reyna, C. \& Brussino, S. (2015). Diferencias de edad $y$ género en comportamiento social, temperamento y regulación emocional en niños argentinos. Acta Colombiana de Psicología, 18(2), 51-64. https://doi.org/10.14718/ACP.2015.18.2.5

Rodríguez, M. C. (2010). Factores personales y familiares asociados a los problemas de comportamiento en niños. Estudos de Psicologia Campinas, 27 (84), 437-447.

Sardón-Prado, O., Morera, G., Herdman, M., Moreno Galdó, A., Pérez-Yarza, E. G., ... Rajmil, L. (2008). Versión española del tapqol: calidad de vida relacionada con la salud en niños de 3 meses a 5 años. Anales de Pediatría, 68(5), 420-424. https://doi.org/10.1157/13120037

Schepers, S. A., van Oers, H. A., Maurice-Stam, H., Huisman, J Verhaak, C. M., ..., Haverman, L. (2017). Health related quality of life in Dutch infants, toddlers, and young children. Healt Quality Life Outcomes, 15(1), 81. https://doi. org/10.1186/s12955-017-0654-4

Tay, C., Jalaludin, M., Low, W., \& Lim, C. (2015). Cross-cultural adaptation and validation of the Malay language version of the Tzo-AzL Preschool Children Quality of Life questionnaire: A health-related quality of life instrument for preschool children. Journal of Child Health Care, 19(2), 167-181. https://doi. org/10.1177/1367493513503583

Urzúa, A., Cortés, E., Vega, S., Prieto, L., \& Tapia, K. (2009). Propiedades psicométricas del cuestionario de auto reporte de la calidad de vida KIDSCREEN-27 en adolescentes chilenos. Terapia Psicológica, 27(1), 83-92.

Urzúa, A., Méndez, F., Acuña, C., \& Astudillo, J. (2010). Calidad de vida relacionada con la salud en edad preescolar. Revista Chilena de Pediatría, 81(2), 128-37. 
Vélez, C., Villada A., Amaya, A. C, \& EslavaSchmalbach, J. (2016). Validación por modelo de Rasch del Cuestionario de Calidad de Vida (PedsQL 4.0 $0^{\circledR}$ ) en niños y adolescentes colombianos. Revista Colombiana de Psiquiatria, 45(3),186-193.

Vinaccia, S. \& Quiceno, J. M. (2010). Calidad de vida relacionada con la salud. Revista Colombiana de Cancerología, 14 (4), 187-188.
Vinaccia, S. \& Quiceno, J. M. (2012). Calidad de vida relacionada con la salud en enfermos crónicos colombianos. Revista Latinoamericana de Psiquiatría, 13(4), 156-163.

WHOQOL Group, (1995). The World Health Organization Quality of life assessment (wHOQOL): Position paper from the world health organization. Social Science and Medicine, 41, 1403-1409. 